\title{
Protée
}

\section{Dépression du spectacle de la sur-nature : réflexions autour du travail de Diane Thater}

\section{Christine Ross}

Volume 28, numéro 3, 2000

Mélancolie entre les arts

URI : https://id.erudit.org/iderudit/030603ar

DOI : https://doi.org/10.7202/030603ar

Aller au sommaire du numéro

Éditeur(s)

Département des arts et lettres - Université du Québec à Chicoutimi

ISSN

0300-3523 (imprimé)

1708-2307 (numérique)

Découvrir la revue

Citer cet article

Ross, C. (2000). Dépression du spectacle de la sur-nature : réflexions autour du travail de Diane Thater. Protée, 28(3), 43-52. https://doi.org/10.7202/030603ar

\section{Résumé de l'article}

La vidéographie intéressée par la question du spectacle pose la question de l'histoire du développement technologique et de son rôle dans la constitution du sujet moderne. Les installations vidéo de Diana Thater doivent être perçues comme une pratique de dépression de la relation (post)moderne entre subjectivité et technologie. L'objectif du présent article est double. D’abord, cerner les possibilités actuelles de mise en scène critique du spectacle. Ensuite, voir en quoi les stratégies esthétiques de dépression de l'image électronique se rapportent aux mutations de la subjectivité contemporaine en cours depuis les années 60 . 


\section{DÉPRESSIO N DU SPECTACLE DE LA SU R-N ATU RE: RÉFLEXIONS AUTOUR DU TRAVAIL DE DIANA THATER}

CHRISTINE ROSS

Dans le cadre de sa participation à la biennale du Carnegie International 1999/ 2000, l'artiste américaine Diana Thater s'appropriait le site même du Botany Hall du Museum of Natural History de Pittsburgh pour y déployer une installation vidéo, visant tout à la fois à articuler et à jeter un regard critique sur la mise en spectacle de la nature. La salle du Botany Hall est une toute petite salle muséologique qui dissémine le savoir botanique par une succession de jardins artificiels creusés dans les murs. L'intervention de Thater consistait à projeter de façon concomitante et continue une multiplicité d'images vidéo silencieuses représentant des dauphins nageant librement dans l'eau. Les projections multiples - contrôlées par une dizaine de projecteurs situés dans différents points de l'espace - avaient pour effet notable de délimiter les images les unes par rapport aux autres, les empêchant de se fondre les unes dans les autres dans un tout panoramique. Une série de stries correspondant aux limites de chaque image territorialisaient ainsi le plafond en divers quadrilatères écraniques, la salle se voyant visuellement mobilisée par une multiplicité kaléidoscopique d'images sans son. Ce qui néanmoins se présentait, d'un premier coup d'œil, comme la nature à l'état sauvage - les dauphins circulant librement dans l'eau - devait peu à peu être admis dans sa dimension cultivée. Ce déplacement, voire cette déception, s'élaborait par la projection d'images d'entraîneurs partageant les mêmes eaux que les mammifères aquatiques. Le spectateur était donc progressivement appelé à réaliser que ces eaux correspondaient en fait à des bassins artificiels d'élevage et d'observation scientifique. C'est alors que la stratification écranique du plafond prenait tout son sens: autant le bassin est-il un territoire qui circonscrit les mouvements des dauphins, autant les projections en territorialisent leur représentation. Ce que l'installation in situ révélait en fin de compte, c'est la spectacularisation de la nature par le musée d'histoire naturelle grâce à l'aménagement des salles et l'exploitation de technologies contemporaines de l'image: les projections vidéo, tels les jardins artificiels creusés dans les murs, sont des petits théâtres qui délimitent, classifient et mesurent l'objet naturel. En d'autres termes, l'œuvre montrait la muséologie comme un mode de connaissance et de diffusion du savoir fondé sur la mise en spectacle de la nature. 
S'engager dans une installation de Diana Thater, c'est entreprendre un travail de deuil par rapport à la notion de progrès, dans la mesure où la nature est représentée comme façonnée par les lois technologiques du spectacle qui non seulement colonisent l'élément naturel à des fins d'observation et de connaissance, mais qui effacent également toute évidence de dé-naturalisation. La force de ce travail tient à sa faculté de mettre en question le rapport que le sujet contemporain entretient avec les technologies du spectacle, plus précisément avec le spectacle de la sur-nature. L'hypothèse que je voudrais développer est la suivante: la vidéographie, lorsqu'elle est exploitée en tant qu'espace d'interaction médiatique, pose la question de l'histoire du développement technologique et de son rôle dans la constitution du sujet moderne. Je dirais même, et ceci est sûrement plus spécifique à l'œuvre de Diana Thater, que l'intermédialité doit être vue comme un moyen de déprimer la relation (post)moderne entre subjectivité et technologie. Mon objectif ici sera donc double.

D'abord, cerner les possibilités actuelles de mise en scène critique du spectacle. Ensuite, voir en quoi les stratégies esthétiques de dépression de l'image électronique se rapportent aux mutations de la subjectivité contemporaine en cours depuis les années 60.

\section{LE SPECTACLE}

En quoi le spectacle est-il problématique? À quoi pourrait correspondre sa mise en visibilité? Quelles sont les stratégies esthétiques aptes à problématiser la technologie du spectacle? Pour tenter de répondre à ces questions, il est crucial de revenir à Guy Debord, un des premiers théoriciens à avoir proposé une réflexion critique sur le spectacle dans son élaboration contemporaine. Ce retour permet de dégager non seulement une des définitions les plus articulées du spectacle, mais aussi les présupposés théoriques qui structurent une telle définition. Comme je chercherai à le démontrer, l'utilité encore actuelle de la notion debordienne tient à sa compréhension du spectacle en termes de séparation.
Mais cette utilité a aussi ses limites que l'œuvre de Thater permet de dégager.

Dans La Société du spectacle (1967), Debord définit la société moderne comme une organisation sociale qui a perdu sa valeur de société en ce que le spectacle est précisément ce qui mine le lien social qui soustend le tenir-ensemble propre à toute communauté. Dès le paragraphe 25, il écrit: «La séparation est l'alpha et l'oméga du spectacle "1. Cet effet de séparation - la fragmentation du lien social et l'extériorisation du spectacle par rapport au sujet - provient d'une médiation accrue du réel, d'une victoire de l'apparence sur la réalité, causée non pas tant par la présence de plus en plus envahissante des images que par les développements du capitalisme et sa constitution d'un sujet en état continu de contemplation et de méconnaissance. Sa caractérisation des effets de séparation du spectacle est clairement énoncée dans le passage suivant et montre à quel point elle se rattache au constat d'une commodification intensifiée de la vie quotidienne:

L'aliénation du spectateur au profit de l'objet contemplé (qui est le résultat de sa propre activité inconsciente) s'exprime ainsi: plus il contemple, moins il vit; plus il accepte de se reconnaître dans les images dominantes $d u$ besoin, moins il comprend sa propre existence et son propre désir. L'extériorité du spectacle par rapport à l'homme agissant apparaît en ce que ses propres gestes ne sont plus à lui, mais à un autre qui les lui représente. ${ }^{2}$

Pour le théoricien donc, le sujet existe à l'extérieur de lui-même, c'est-à-dire séparé de ses propres gestes et désirs qui n'existent que représentés par les images et les objets qui l'entourent. Debord a une compréhension marxiste de cet état d'extériorisation, puisqu'il est perçu comme dérivant du développement du mode capitaliste de production qui sépare le travailleur de son produit à travers la spécialisation des tâches et la parcellisation des gestes ${ }^{3}$. Il dérive également de la commodification développée des objets qui fait du sujet un contemplateur passif de la réalité en le nourrissant de faux besoins et de faux désirs. En d'autres termes, dans une société du spectacle, l'individu absorbe et est absorbé par des 
images qui semblent lui conférer cohérence et unité, mais qui en fait le désunifient. Pour réactualiser ce constat en termes de théorie de l'identification, on peut dire que l'identité du sujet contemporain prend forme par ce que Kaja Silverman désigne comme une identification idiopathique. Ceci signifie que le sujet contemplatif en est un qui se méconnaît de façon répétitive dans l'image, comme dans l'expérience du stade du miroir décrite par Lacan où l'enfant incorpore l'autre comme s'il était le moi, confondant besoins et désirs, idéalité et réalité. Une telle passivité d'adhésion aux images contemporaines atteste du fait que les images spectaculaires "sont facilement incorporées» et qu'elles fournissent au sujet un sens illusoire de cohérence, d'unité et de maîtrise ${ }^{4}$, même si la méconnaissance est une division de lui-même.

Le spectacle sous-tend donc un effet d'absorption par l'image pour autant qu'on comprenne que cet effet correspond à une séparation du sujet par rapport à son environnement (le sujet comme transcendance) et à une négation du sujet désirant, divisé par son entrée dans l'ordre symbolique (le sujet comme conflit). Le spectacle est une réalité qui confère au sujet une subjectivité transcendante et unifiée, remédiant ainsi - de façon temporaire tout au moins au manque constitutif du sujet moderne. La pertinence encore actuelle d'une telle définition force à être attentif aux passages où Debord élabore une critique antioculaire du spectacle puisqu'il est loin d'être sûr qu'une telle critique puisse encore tenir aujourd'hui. Le spectacle est une organisation sociale qui prend forme dans une période où la vision est devenue le sens privilégié de la perception: remplaçant le toucher - sens du lien et du contact-, la vision a ceci de problématique qu'elle favoriserait la passivité perceptive, l'illusion et la méconnaissance. Ce qui est significatif à propos de cette perspective antivisuelle, c'est qu'elle introduit un paradoxe important dans La Société du spectacle, laquelle peut se résumer ainsi: Debord se positionne comme un observateur apte à reconnaître le spectacle, malgré le triomphe de la vue et au détriment de la perte du toucher, même si le spectacle est, par définition, un processus impossible à reconnaître, puisqu'il découle précisément de la méconnaissance réitérée du sujet qui croit voir dans l'image ou dans l'objet des produits de sa propre activité, alors que ceux-ci résultent de la logique capitaliste de commodification. Ce premier paradoxe est relié à un deuxième problème méthodologique, qui consiste à définir le spectacle comme une organisation unifiée - une «vérité générale»-, alors qu'il se rapporte intrinsèquement à un processus de fragmentation, de parcellisation et d'extériorisation. Debord insiste:

Le concept de spectacle unifie et explique une grande diversité de phénomènes apparents. Leurs diversités et contrastes sont les apparences de cette apparence organisée socialement, qui doit être reconnue dans sa vérité générale. [...] L'origine du spectacle est la perte de l'unité du monde, et l'expansion gigantesque $d u$ spectacle moderne exprime la totalité de cette perte. ${ }^{5}$

Qu'est-ce à dire sinon que, pour Debord, le spectacle est paradoxalement une désunification du réel par la vision et une totalité unifiée qui peut (précisément parce qu'elle est unifiée) devenir visible par un regard critique. La théorie debordienne est donc aussi totalisante - aussi négatrice de la désunification - que le spectacle qu'elle cherche à miner. Par ailleurs, le démasquage des illusions du spectacle qu'elle souhaite opérer est une politique rédemptrice de reconnaissance qui oublie un des premiers préceptes de Debord, à savoir que le spectacle est une société façonnée par la méconnaissance.

La question qui s'impose est donc celle-ci: comment adopter la catégorie du spectacle pour des fins critiques sans réitérer le totalitarisme et l'antioculisme inhérents au modèle debordien? Quelques décennies après La Société du spectacle, la critique du spectacle semble plutôt passer par une re-pensée de la vision que par sa pure et simple condamnation. Chez Diana Thater, cette re-pensée de la vision exprime la nécessité de troubler les fantasmes de transcendance et de désaveu du sujet contemporain, et articule une critique de la vision monoculaire telle qu'elle s'est développée depuis le développement quattrocentiste de la perspective ( «nous n’avons pas réussi, écrit-elle, à déconstruire un 
monde qui [...] autorise visuellement la vision monoculaire de l'œil immobile» $\left.{ }^{6}\right)$. Comme le soutient l'historienne d'art Amelia Jones dans son examen du body art, une critique du spectacle ne présuppose pas nécessairement un refus ou une dénégation du plaisir visuel ${ }^{7}$. En fait, discréditer le plaisir visuel signifie dénier le rôle du corps dans la perception, sa part physiologique et sensuelle, tout comme cela signifie méconnaitre l'impact du spectacle sur la perception - le fait qu'une critique du spectacle ne peut s'articuler que par un sujet percevant lui-même marqué par le spectacle. Les recherches menées par Jonathan Crary sur les origines dix-neuvièmistes du spectacle ont montré comment la perception est un acte discipliné et normalisé autant par les discours scientifiques dominants que par la technologie optique développée non seulement pour mesurer la perception, mais aussi pour divertir le spectateur en termes de plaisir visuel (pensons ici au kaléidoscope, à l'appareil photographique et à la cinématographie). Comme le maintient la théoricienne de cinéma Linda Williams, la perception moderne se matérialise par l'intégration de plaisirs visuels issus de la culture populaire de divertissement. Il importe de reconnaitre ce processus si nous voulons comprendre quelque chose non seulement à la perception contemporaine, mais aussi au potentiel de perceptibilité critique du sujet:

"Le rapt, la fascination stupide»: ceci correspond certainement à une apte formulation de ce qui provoque la présente anxiété exprimée à propos de la culture de masse visuelle contemporaine et les dangers de la capture visuelle. Nous devons néanmoins nous questionner sur l'utilité actuelle d'une telle caractérisation de la soumission spectatorielle à l'image visuelle du film. Le problème relié à une telle formulation se situe dans le fait qu'elle érige une opposition entre les plaisirs sensuels de la vision et l'abstraction de la pensée critique - comme si la pensée ne pouvait jamais s'articuler par et à travers le corps. En accusant les spectateurs de participer à l'excès à la fois du visuel et du corporel, elle réduit cet excès à une pornographie "essentielle» qui ne fait qu'avilir la vision en tant que telle. La vision, spécialement la vision qui engage le corps dans une réponse viscérale, est stigmatisée soit comme étant une forme abusive de pouvoir ou au contraire comme une forme de passivité. 8
Pour ce qui est de la croyance qu'a Debord en la possibilité de reconnaitre le spectacle, ses paradoxes révèlent que celui-ci n'est pas quelque chose que l'on peut espérer reconnaître pour la simple raison qu'il n'est pas plus unifié que le sujet qui le contemple. En d'autres termes, si pour Debord «le concept de spectacle unifie et explique une grande diversité de phénomènes apparents", cette unité ne peut être que temporaire si l'on tient compte du fait que la subjectivité est toujours une subjectivité en procès, formée par une série d'identifications et de désidentifications conscientes et inconscientes, interdépendantes bien que souvent contradictoires. La tentative de Debord de reconnaître l'unité supposée du spectacle est loin d'être la réponse la plus satisfaisante à son développement. Comme je souhaite le démontrer, les installations de Diana Thater n'opèrent pas tant une révélation, une reconnaissance ou une distanciation du spectacle qu'une intermédialité qui favorise au contraire l'identification, la projection et la proximité. L'objectif est de ne pas retirer le spectateur du domaine du spectacle, mais d'inscrire son activité visuelle dans ce que j'appellerais la matérialité de la projection. L'intermédia est exploité de façon telle que l'installation devient un espace d'immersion partielle du spectateur, qui articule des mécanismes structurels et formels pour questionner le processus d'unification du spectacle et pour créer une brèche dans la définition lacanienne du sujet, selon laquelle le sujet n'est sujet que dans la mesure où il est toujours un être regardé, «dans le spectacle du monde» ${ }^{9}$. Si, pour Lacan et Debord, le spectacle est une totalité (il englobe le sujet), l'intermédia conçu par Thater est plutôt une question sur la totalité, c'est-à-dire sur les limites de la victoire de l'apparence sur la réalité et les possibilités du sujet à faire le deuil du spectacle.

\section{LA SUR-NATURE}

Lorsqu'on entre dans une installation de Diana Thater, on circule dans un espace intermédia qui met en scène le spectacle de ce que Lucien Sfez a récemment désigné comme le spectacle de la sur- 
nature - la croyance utopique (caractéristique de la fin du 20e siècle) en notre capacité de prédire, de contrôler, de conquérir et de perfectionner la nature par la technologie (tels l'ingénierie génétique, la robotique, les biotechnologies de reproduction, l'écotechnologie antipollution, la visualisation numérique, la pharmacologie et les appareils de la body-fitness), de façon à assurer notre survie ${ }^{10}$. Ses mises en scène prennent habituellement la forme de projections filmiques ou vidéo d'images de la nature - paysages de fleurs multicolores, chevaux sauvages courant librement dans une campagne rustique, zèbres, chevaux ou singes faisant des prouesses acrobatiques devant la caméra, dauphins nageant ouvertement dans l'eau -, à travers lesquelles le spectateur est invité à circuler. Très rapidement, le spectateur devient conscient qu'il regarde une nature recréée: les fleurs sont des fleurs cultivées, les animaux sauvages sont des acteurs professionnels filmés par une équipe de tournage souvent visible dans l'image, les dauphins circulent dans un bassin d'eau territorialisé, les paysages naturels sont des parcs à thème aménagés comme le Medieval Times Buena Parc et le L.A. County Arboratum en Californie. Ici, les technologies de l'image (le film, la vidéo et je dirais même la peinture) s'allient aux éco-technologies pour constituer une surnature en termes de spectacle. Les installations de Thater montrent comment la sur-nature dépend non seulement des bio- et éco-technologies pour s'élaborer, mais aussi des technologies de l'image qui la représentent et assurent son déploiement en termes de spectacle, un spectacle défini ici comme un système discursif visuel constitutif d'un désir de transcendance du sujet par rapport au corps, à l'autre, à l'histoire, à l'environnement. En d'autres termes, elles révèlent en quoi la sur-nature, mise en spectacle qui présuppose prévisibilité et contrôle du corps et de la planète, est une négation de la mortalité, un désir de transcender la maladie et d'accéder à un réel dénué de toute forme d'incertitude, de manque et de contingence. Pour ce faire, et c'est ce qui les distingue des projets scientifiques décrits par Lucien Sfez (entre autres, le Projet du Génome Humain et Biosphère II ${ }^{11}$ ), elles intègrent la dépression à même le spectacle. Le spectacle de la sur-nature ou la sur-nature comme spectacle s'y déploie mais pour être potentiellement déprimé par diverses stratégies esthétiques, telles l'absence de son, l'intégration des équipes d'entraînement ou de tournage à même l'image, la mise en évidence des appareils de diffusion (moniteurs, magnétoscopes) et du filage dans l'espace. La pertinence de ce travail se situe dans son élaboration d'une série de stratégies intermédiatiques, qui repense le rapport du spectateur au spectacle de la sur-nature, afin de lui permettre de voir comment et en quoi sa subjectivité se constitue à même le «sauvage réinventé" si caractéristique de l'utopie de la surnature. Le spectacle est donc représenté non pas comme un phénomène en soi (à condamner ou à célébrer), mais comme un processus constitutif de la subjectivité contemporaine.

\section{L'INSUFFISANCE DU VOIR}

ET LA PERTE DU CORPS PERCEPTIBLE

Dans son étude sur Marcel Duchamp, David Joselit souligne comment, à partir de la fin du 19 e siècle, un certain pan de l'art moderne a sonné le glas de la représentation métaphorique du corps pour en représenter sa commodification ${ }^{12}$. Dès l'Olympia (1865) de Manet, l'art établit une équivalence entre le corps, le signe et le marché. Ici, la femme - dont le sens oscille, comme l'a démontré T.J. Clark ${ }^{13}$, sans jamais se fixer, entre le nu idéal et la prostituée des faubourgs parisiens - n'est pas comme une commodité, elle est une commodité, perdant sa valeur et de nature et d'utilité pour assurer, en tant que valeur d'échange, les relations entre hommes. Transformé en devise, le corps n'est plus perceptible, c'est-à-dire qu'il se retire comme corps naturel: sémantiquement colonisé, il n'existe plus qu'à travers sa dimension sémiotique de marchandise. Le Grand Verre (1915-1923) de Duchamp s'inscrit dans cette même perte de visibilité du corps. Articulant deux espaces séparés qui isolent les célibataires de la mariée, le Verre est une poésie de la perte par le délai sans fin de consommation érotique qu'il met en œuvre. Le corps, chez 
Duchamp, a ingéré la logique de la consommation, du délai, de l'échange infini rattaché à la valeur d'échange. Mais s'il y a effectivement perte du corps moderne à la vision, un constat développé par Joselit mais initialement formulé par Rosalind Krauss dans son examen des œuvres cubistes de Picasso, n'y a-t-il pas corrélativement perte des facultés perceptives empêchant le sujet de reconnaître le processus de commodification du corps? Dans son étude sur les arcades parisiennes, Walter Benjamin stipule - dès les années 20 et 30 - que le sujet moderne a perdu sa faculté d'aperception et que celle-ci a progressivement fait place à une forme d'anesthésie de la conscience ${ }^{14}$. Pour parer aux agressions de la modernité urbanisante (massification, industrialisation, intensification sensorielle), le sujet a appris à engourdir ses facultés perceptives et mnémoniques en faisant de plus en plus appel à différentes formes d'intoxication, tels que l'opium et les divertissements de masse incluant le cinéma, la foire publique et les magasins à rayons. Si la modernité se définit comme une perte du corps naturel, c'est qu'elle engendre également une perte incarnée de la perception consciente. Cette perte - qui n'est pas nécessairement négative ou pleinement achevée-, je la verrais comme une insuffisance $d u$ voir.

Lorsqu'on pense à l'ensemble des procédés prothétiques aujourd'hui disponibles pour augmenter les capacités oculomotrices de l'être humain, on réalise à quel point le développement de la vision est un champ d'étude extrêmement actif dans le domaine scientifique. Endoscopie, laparoscopie et tomographie assistée par ordinateur, échographie, résonance magnétique, microscopes électroniques, télescopes satellites, caméras de surveillance, technologies d'identification criminelle, scanners à fonctions multiples: ces systèmes de visualisation élaborent une panoplie d'images qui repoussent constamment les frontières du perceptible. Fait significatif néanmoins, les recherches récentes de Paula Treichler, de Lisa Cartwright et de Sarah Kember, entre autres, montrent que cette capacité à élargir le champ du visible entraîne avec elle une anxiété visuelle grandissante chez les experts, dans la mesure où la compétence requise pour lire et interpréter ces images fait de plus en plus problème ${ }^{15}$. Insuffisance et amélioration du voir sont des données inséparables. Les capacités de l'œil semblent pouvoir être améliorées de façon infinie, mais elles ne répondent pas toujours à la machine. Cette insuffisance est aussi constatée par la neurobiologie, les sciences cognitives et la psychanalyse où la recherche sur les déficits d'attention, les troubles de sommeil et la dépression occupent un champ de plus en plus important de l'enquête scientifique. Des publications sur l'aveuglement non intentionnel, sur l'impossibilité d'une perception (consciente) sans attention, sur l'altération des facultés perceptives du sujet dépressif, sur l'impact des images mentales sur la vision ne sont que quelques exemples de discours scientifiques préoccupés par la difficulté de définir la perception comme simple enregistrement du monde. La question que je voudrais soulever ici est la suivante: si le corps est "en retard" par rapport à la machine optique, comment profiter de ce retard, de cette insuffisance du voir pour mettre en question le mythe si clairement énoncé par Donna Haraway dans son examen des technologies modernes de visualisation: «le mythe de pouvoir tout voir à partir de nulle part» 16 ?

L'élaboration de l'insuffisance perceptive en est une -c'est l'hypothèse que je tente de développer iciqui critique non seulement le mythe de "pouvoir tout voir", mais l'identité unifiée et transcendante qu'un tel mythe présuppose.

Diana Thater explique en ces termes le questionnement que je cherche à décrire ici:

Entre les types de caméras et l'équipement de projection utilisés, je veux que le spectateur puisse voir simultanément des espaces plats et profonds, ainsi que la place à partir de laquelle il voit et l'espace dans lequel il regarde. [...] une peinture de Bridget Riley [...] n'est pas une entrée dans l'espace profond derrière mais en face de la toile. De la même façon, mon travail a comme sujet l'écran plat et l'espace réel qui se tient devant l'écran en opposition à l'espace illusionniste situé à l'intérieur de l'image. 17 
Regarder à partir d'un espace et dans un espace simultanément, entrer à la fois dans l'espace profond derrière et devant l'image. Une telle simultanéité est mise en marche, entre autres, par l'installation des moniteurs, magnétoscopes, projecteurs, prises de courant et filage sur le plancher du site, qui force le spectateur à prendre conscience de la technologie de son regard. Elle est aussi mise en œuvre par la structuration multiécranique de l'espace qui amène le spectateur à prendre conscience de son inscription dans un espace physique territorialisé à partir duquel il interagit, par son propre corps, avec l'espace imaginaire dans l'image.

Ainsi, avec l'installation The Best Sense is the Nonsense (1999), Diana Thater propose un espace composé de deux écrans de projection et d'un moniteur vidéo placés l'un devant l'autre, chacun transmettant des images de zèbres qui font différentes acrobaties à l'aide d'un entraîneur. Des images filmiques transférées sur vidéo sont projetées sur le mur du fond, le mur le plus éloigné du site. Ces images à haute résolution (si on les compare aux images vidéo de l'écran du milieu et du moniteur) sont paradoxalement les moins visibles et les moins facilement accessibles. Par ailleurs, elles donnent lieu à des séquences où les rayures de l'animal, saisies en gros plan, inondent l'écran, ce qui occasionne un aplatissement de l'image qui bloque l'entrée imaginaire du spectateur dans l'image. Le spectateur est ramené à la surface de l'image, c'est-à-dire, plus précisément, à la frontière qui sépare l'extérieur de l'intérieur, l'espace physique de la salle de l'espace illusionniste de l'image.

L'installation est également composée de projecteurs de lumière qui activent, lorsque le spectateur se trouve près de l'écran, la projection de son ombre sur l'écran. Cette projection est opérée de façon telle que, dans le cas de l'écran du fond, elle réduit l'échelle du spectateur et que cette échelle devient incompatible avec celles de l'entraineur et de l'animal dans l'image. Cette incompatibilité appuie le blocage opéré par les rayures: le spectateur se voit à nouveau refuser son entrée dans l'espace imaginaire de l'image. La projection vidéo du centre élabore un effet de blocage similaire mais qui s'articule cette fois par une augmentation de l'échelle du spectateur, celleci devenant démesurément grande par rapport aux corps représentés dans l'image. On peut commencer à entrevoir le type de relation que cet espace intermédia exerce entre le spectateur et l'image. Les seuls moments où le spectateur entre dans l'espace illusionniste de l'image sont les moments où il est à distance. Lorsqu'il est proche et que l'image devient accessible, son entrée sera bloquée. Par ailleurs, les images filmiques à haute résolution sont les moins accessibles de l'installation. Ce questionnement du rapport entre le proche et le lointain, entre l'entrée dans et le blocage par l'image, est renforcé par l'installation des écrans un derrière l'autre dans l'espace. Cette succession de plans introduit le spectateur dans un espace proprement pictural, un espace constitué d'un avant-plan, d'un plan médian et d'un arrière-plan, qui est ici inversé puisque la récession des orthogonales s'élabore à partir de l'arrière vers l'avant contrairement au système perspectiviste hérité de la Renaissance, où les orthogonales se rejoignent au point de fuite situé au centre de la ligne d'horizon. En d'autres termes, les orthogonales ici se rejoignent au point de fuite situé à l'avant-plan de l'espace, c'est-à-dire au point où se situe traditionnellement le point de vue.

Ce que l'intermédialité film-vidéo-peinture instaure en fin de compte, c'est une série d'oscillations entre distance et proximité, espace imaginaire et espace physique, point de vue et point de fuite, avant et arrière, profondeur et surface, une série d'oscillations qui s'élaborent par notre déplacement dans l'espace et qui ne cessent de déprimer notre horizon d'attente. Suis-je proche d'une image qui se refuse à moi? Suis-je loin? Où suis-je si j'occupe le point de fuite dans lequel, théoriquement, je disparais? Où commence l'espace illusionniste, où se termine l'espace physique? Où s'arrête le spectacle? Où débute-t-il? En fait, dès que la question de la spatialité du spectacle naturel est posée, c'est celle de la représentation qui émerge, conséquemment celle de 
la subjectivité, c'est-à-dire celle de la mise en spectacle pour les fins d'une subjectivité.

Qui dit intermédia chez Thater dit également intermédialité de l'image. Je ne fais pas tant référence ici aux transferts film-vidéo, qui sont fréquents dans l'œuvre de Thater, qu'au travail de répétition de l'image, plus précisément le montage qu'exploite Thater à travers ses installations, opération qui consiste à introduire une séquence narrative pour ensuite la couper et la reproduire. L'installation The Best Space is the Deep Space (1999), par exemple, présente sur trois moniteurs et avec un léger décalage, mais toujours de façon répétée, une même séquence d'une dizaine de secondes mettant en scène un cheval filmé avec son entraîneur qui le guide dans sa génuflexion. Thater exploite ici, comme dans la plupart de ses œuvres, la capacité de reproduction et de recyclage de l'image vidéographique pour introduire progressivement un élément de tragédie dans le spectacle. La répétition de la génuflexion rend manifeste la façon dont la bio-technologie façonne, colonise et réorganise la nature, ici le cheval de cirque. Transmis de façon répétée et recyclée, intermédiatisé d'un écran à l'autre et d'une séquence à l'autre, le façonnement devient manifeste dans son instrumentalité, c'est-à-dire comme façonnement. En d'autres termes, les images du cheval en génuflexion sont toujours significativement en train de mourir ou d'être recyclées pour désigner la pulsion de mort à l'œuvre dans toute sur-nature, une désignation renforcée par l'absence de son et l'exploitation du ralenti. Apparaissant, toujours sur le seuil d'être coupé et reproduit comme même, évanescent au niveau sonore tout au moins, le spectacle est une pulsion de mort.

Les installations offrent en cela une lecture du progrès en tant que désintégration. Thater explore l'image comme Walter Benjamin utilise les arcades parisiennes du 19e siècle transformées en ruine après l'arrivée du magasin à rayons, comme une mémoire collective qui manifeste notre inhabilité à penser le progrès autrement que dans une logique de production effrénée de mondes de rêve, de spectacles, toujours à être abandonnés et remplacés par de nouveaux ${ }^{18}$. Ce faisant, par leur intermédialité, les installations mettent également en cause le discours du progrès technologique selon lequel les images numériques dépassent les images électroniques qui dépasseraient les images picturales. Chez Thater, la peinture, le film et la vidéo sont des quasi-ruines capables d'évoquer notre besoin de nouveaux spectacles pour asseoir notre subjectivité. L'intermédia est en cela un acte de réminiscence, un moyen de «saisir la construction de l'histoire en tant que telle ${ }^{19}$, comme une mise en spectacle de la nature qui nous confirme comme sujets.

\section{LA DÉPRESSION}

Lorsqu'on examine le travail de Diana Thater, la catégorie de la nature est maintenue pour penser sa perte et tenter de la réarticuler autrement. Les stratégies esthétiques se déploient de façon à briser les dimensions métaphoriques et métonymiques de la représentation de la nature. En insérant les modalités d'enregistrement des images (équipe de tournage) dans l'image, en rendant manifestes les moyens de diffusion des images (magnétoscope et filage) dans l'espace de l'installation, en exploitant le cycle perpétuel du ralenti et de la répétition par lequel la nature perd son identité de nature, en indiquant l'effet de cadrage des images, ses paysages et animaux ne sont plus comme la nature ou de nature, mais une nature-spectacle commodifiée, une sur-nature. Ses installations sont donc orchestrées de façon à rendre manifeste le processus de mise en spectacle de la nature, son existence de plus en plus prégnante en tant que spectacle, mais elles sont aussi un site de deuil où le spectacle est électroniquement déprimé. Le spectacle est représenté - mis en scène et situé quelque peu à distance - seulement quand le spectateur accepte de voir et de participer à la dépression du spectacle. S'il accepte, s'il a des chances d'accepter ce jeu, c'est qu'il est un être déprimé ou en voie de l'être.

Selon le sociologue Alain Ehrenberg, la dépression est «révélatrice des mutations de l'individualité à la fin du XXe siècle» ${ }^{20}$. En cela, elle définit le sujet 
contemporain, le spectateur même des installations de Diana Thater. Maladie de la responsabilité dominée par le sentiment d'insuffisance, elle arrive dès les années 60 , période où les normes de socialisation fondées sur la loi du père, la discipline, le sens de la culpabilité et de l'interdit (menace de la castration) commencent à être remplacées par des normes de performance, d'initiative, de flexibilité, de motivation et de communication. Enjoignant l'individu à «devenir lui-même» et à «choisir sa vie», ces nouvelles normes sont constitutives d'un individu qui se développe non plus selon les paramètres freudiens ou lacaniens du permis et du défendu, ou encore de la docilité telle que définie par le taylorisme ou le fordisme, mais selon les paramètres du possible et de l'impossible à la base non seulement de la culture entrepreneuriale mais aussi de la culture plastique surnaturelle du body-building, du face-lifting et du bodyfitness. La dépression résulte de l'incapacité du sujet à répondre à cette demande de mouvement permanent, où «aucune loi morale ni aucune tradition nous indiquent du dehors qui nous devons être et comment nous devons nous conduire» 21 , et où l'individu idéal doit faire appel à ses propres ressorts internes pour se constituer. Ehrenberg:

Hypothèse 1: la dépression nous instruit sur notre expérience actuelle de la personne, car elle est la pathologie d'une société où la norme n'est plus fondée sur la culpabilité et la discipline mais sur la responsabilité et l'initiative. L'individu est confronté à une pathologie de l'insuffisance plus qu'à une maladie de la faute, à l'univers du dysfonctionnement plus qu'à celui de la loi. [...]

Hypothèse 2: le succès de la dépression repose sur le déclin de la référence au conflit (qui permet de maintenir un écart entre ce qui est possible et ce qui est permis) sur laquelle s'est construite la notion de sujet que la fin du XIXe siècle nous a léguée. L'histoire psychiatrique de la dépression est caractérisée par la difficulté à en définir le sujet. [...] Comme la névrose guettait l'individu divisé par ses conflits, déchiré par un partage entre ce qui est permis et ce qui est défendu, la dépression menace un individu apparemment émancipé des interdits, mais certainement déchiré par un partage entre le possible et l'impossible. Si la néurose est un drame de la culpabilité, la dépression est la tragédie de l'insuffisance. Elle est l'ombre familière de l'homme sans guide, fatigué d'entreprendre de devenir seulement lui-même et tenter de se soutenir jusqu'à la compulsion par des produits ou des comportements. 22

La dépression résulte de l'insécurité identitaire, des sentiments de l'insuffisance et de la fatigue d'être soimême, le repli dépressif constituant une «attitude protectrice de retrait», qui permet la survie du sujet quand celui-ci n'a plus la faculté de lutter ${ }^{23}$. Lorsque l'art contemporain repense la vision et situe le spectateur de façon à ce qu'il entreprenne un travail de deuil par rapport au spectacle, au corps, à la nature, au progrès technologique, lorsqu'il se veut critique et que, pour ce faire, il déploie des stratégies d'insuffisance de l'image, c'est qu'il a pris conscience de l'insuffisance du voir contemporain - une insuffisance reliée, entre autres, à la subjectivité même du spectateur contemporain sujet à la dépression, performatif mais insuffisant, plein d'initiative mais fatigué, responsable mais anesthésié, identifié mais désidentifié. Ces états peuvent sembler négatifs mais peuvent aussi être explorés, comme c'est le cas chez Thater, comme productifs puisqu'ils sont une critique de la sur-nature. Ehrenberg définit la dépression comme «une pathologie du temps (le déprimé est sans avenir) et une pathologie de la motivation (le déprimé est sans énergie, son mouvement est ralenti, et sa parole lente)» 24 . C'est précisément cette définition de la dépression qui est à l'œuvre dans les installations de Thater: l'intermédia prive peu à peu le spectacle (par le recyclage, la répétition et le déplacement du spectateur entre les médias) de ses trois principaux outils de conviction: la projectabilité dans le futur par l'oubli du passé, le pouvoir de communication et le pouvoir de motivation. Thater pathologise les médias en élaborant une dépression technologique - l'image se répète, les médias ont été vidés de leur dimension sonore, le spectateur accède à une image qui déçoit son expectative et fait osciller les points de référence par lesquels il constitue sa subjectivité et son positionnement dans l'espace, le site le situe au point de fuite où, théoriquement, il disparaît. La sur-nature ne peut plus alors fonctionner comme une garantie de 
sa continuité et de sa capacité à transcender l'environnement. Comme le soutient Ehrenberg, la dépression, une des principales pathologies de notre temps, est l'envers exact de nos normes de socialisation de performativité, d'affranchissement, de responsabilité, d'initiative et de flexibilité. En cela, elle est "la contrepartie du déploiement de [l']énergie» du sujet contemporain ${ }^{25}$. Lorsqu'elle déprime les images, l'intermédialité offre au sujet un garde-fou qui l'invite à repenser ses limites par rapport au spectacle actuel qu'est celui de la sur-nature.

\section{N O TES}

1. G. Debord, La Société du spectacle, Paris, Éd. Gallimard, 1967, §25.

2. Ibid., $\$ 30$.

3. Ibid., $\$ 26$.

4. K. Silverman, The Threshold of the Visible World, New York, Routledge, 1996, p. 22.

5. Debord, op. cit., $\S 10$.

6. D. Thater, "Skin Deep", dans The Best Animals are the Flat Animals - The Best Space is the Deep Space, Los Angeles, MAK Center for Art and Architecture, 1999, p. 35. Notre traduction.

7. A. Jones, Body Art: Performing the Subject, Minneapolis, University of Minnesota Press, 1998.

8. L. Williams, «Discipline and Distraction: Psycho, Visual Culture, and Postmodern Cinema», dans J. C. Rowe (sous la dir. de), «Culture» and the Problem of the Disciplines, New York, Columbia University Press, 1998, p.91. Notre traduction.

9. J. Lacan, Les Quatre Concepts fondamentaux de la psychanalyse, Paris, Éd. du Seuil, [1964] 1973, p. 121.

10. L. Sfez, La Santé parfaite: critique d'une nouvelle utopie, Paris, Éd. du Seuil, 1995

11. Le Projet du Génome Humain est un projet international qui vise à cartographier et à séquencer la totalité des gènes humains et qui promet de nous donner avec précision les origines génétiques de l'ensemble des maladies de façon à ce qu'on puisse éventuellement supprimer, par manipulation génétique, les mauvais gènes qui menacent la santé de l'individu. Biosphere II (1991-1993) est un projet américain qui consistait à construire un modèle réduit de la biosphère terrienne en plaçant sous d'immenses hangars de verre, pendant une période de deux ans, les cinq principaux biomes de l'humanité, 3000 espèces d'animaux et de plantes et huit humains. Bien que le projet ait échoué, l'objectif principal était de traiter les problèmes de l'environnement et de construire un modèle qui servirait de prototype pour des colonies dans l'espace, à la lumière de la prophétie que le système solaire va se détruire dans 300 millions d'années et que l'humain aura bientôt démoli l'équilibre écologique de la planète. 12. D. Joselit, Infinite Regress: Marcel Duchamp 1910-1941, Cambridge (Mass.), The MIT Press, 1998.

13. T. J. Clark, "Preliminaries to a possible treatment of "Olympia" in 1865", Screen, vol. XXI, n 1 , printemps 1980, p. 18-41.

14. Voir S. Buck-Morss, The Dialectics of Seeing: Walter Benjamin and the Arcades Project, Cambridge (Mass.), The MIT Press, 1995.

15. Voir, entre autres, P. Treichler et L. Cartwright (sous la dir. de), Imaging technologies, Inscribing Science, $\mathrm{n}^{\circ}$ spécial de Camera Obscura (28), 1992; et S. Kember, Visual Anxiety: Photography, New Technologies and Subjectivity, Manchester, Manchester University Press, 1998.

16. D. J. Haraway, «Situated Knowledges: The Science Question in Feminism and the Privilege of Partial Perspective", dans Simians, Cyborgs, and Women: The Reinvention of Nature, New York, Routledge, 1991, p. 189.

17. Thater, entretien avec le "Education Department", New York, Museum of Modern Art, 1998. Notre traduction.

18. S. Buck-Morss, déjà cité à la note 14 .

19. W. Benjamin, cité par S. Buck-Morss, op. cit., p. 78.

20. A. Ehrenberg, La Fatigue de soi : dépression et société, Paris, Éd. Odile Jacob, 1998, p. 10.

21. Ibid., p. 14

22. Ibid., p. 15-17.

23. Ibid., p. 183.

24. Ibid., p. 250.

25. Ibid. 